\title{
Altered microbiota associated with abnormal humoral immune responses to commensal organisms in enthesitis-related arthritis
}

\author{
Matthew L Stoll ${ }^{* *}$, Ranjit Kumar ${ }^{2}$, Casey D Morrow ${ }^{3}$, Elliot J Lefkowitz ${ }^{4}$, Xiangqin Cui ${ }^{5}$, Anna Genin ${ }^{1}$, Randy Q Cron ${ }^{1}$
} and Charles O Elson ${ }^{6}$

\begin{abstract}
Introduction: Prior studies have established altered microbiota and immunologic reactivity to enteric commensal organisms in inflammatory bowel disease (IBD). Since intestinal inflammation is present in a subset of patients with both pediatric and adult spondyloarthritis (SpA), we hypothesized that SpA patients may also have altered microbiota and immune responsiveness to enteric organisms.

Methods: Stool and blood specimens were collected from children with enthesitis-related arthritis (ERA) and non-inflammatory controls. DNA purified from stool was subject to PCR amplification and sequencing of the variable IV region from the $16 \mathrm{~S}$ rDNA gene. IgA and IgG Enzyme-linked Immunosorbent Assays (ELISAs) were performed on select species of bacteria in most subjects.
\end{abstract}

Results: Twenty-five children with ERA and 13 controls were included. The ERA patients had less Faecalibacterium prausnitzii (3.8\% versus $10 \%, P=0.008)$ and lachnospiraceae family (12 versus $7.0 \%, P=0.020$ ), a statistically significant increase in bifidobacterium $(1.8 \%$ versus $0 \%, P=0.032)$ and a non-statistically significant increase in Bacteroides (21\% versus $11 \%, P=0.150$ ). Akkermansia muciniphila was abundant $(>2 \%)$ in $7 / 27$ ERA patients but none of the controls $(P=0.072$.) Cluster analysis revealed two clusters of ERA patients: Cluster one $(n=8)$ was characterized by high levels of Bacteroides genus, while a second $(n=15)$ cluster had similar levels as the controls. Seven of 17 (41\%) of the ERA subjects in Cluster 2 compared to 0/8 of the subjects in Cluster 1 had abundant Akkermansia muciniphila $(P=0.057)$. Serum $\lg A$ and $\lg G$ antibody levels against $F$. prausnitzii and $B$. fragilis were similar between patients and controls, whereas the two groups showed divergent responses when the fecal relative abundances of F. prausnitzii and Bacteroides were compared individually against IgA antibody levels recognizing F. prausnitzii and B. fragilis, respectively.

Conclusion: The abundance of F. prausnitzii in the stool among patients with ERA is reduced compared to controls, and Bacteroides and A. muciniphila are identified as associative agents in subsets of ERA patients. Differences in the humoral responses to these bacteria may contribute to disease.

\section{Introduction}

Spondyloarthritis (SpA) affects up to $1 \%$ of the population in the United States [1]. Recent genetic advances have shed some light on pathogenesis and have shown important overlap with inflammatory bowel disease (IBD) [2,3]; however, there still remain important environmental

\footnotetext{
* Correspondence: mstoll@peds.uab.edu

'Department of Pediatric Rheumatology, University of Alabama at Birmingham, CPP N 210M, 1600 7th Avenue South, Birmingham, AL 35233, USA

Full list of author information is available at the end of the article
}

components. Given the association between gut inflammation and SpA [4], one potential environmental trigger that has long attracted attention is the microbiota; indeed, the disease is abrogated in an HLA-B27 transgenic rodent model of colitis and spondylitis when the animals are raised in a germ-free environment [5].

Advances in technology, collectively known as nextgeneration sequencing, have dramatically lowered the costs of sequencing and thus permitted collections of vast amounts of data, as many as one billion short sequences, in one run [6]. One of the applications has been the 
assessment of the entire enteric microbiome from multiple individuals. Studies have shown altered microbiota in a variety of inflammatory conditions, including IBD, rheumatoid arthritis (RA), type I diabetes, and celiac disease [7]. Using a variety of molecular approaches, Stebbings et al. [8] demonstrated possible decreases in the Bacteroides-Provotella and Clostridium leptum groups, with an increase in Bifidobacterium among ankylosing spondylitis (AS) patients compared to controls, although the overall differences were not statistically significant [8]. As this study did not use next generation sequencing technology, the nature of the microbiota in SpA patients has yet to be fully assessed.

Intestinal bacteria need not be present in abnormal quantities to trigger arthritis; it is also possible that a pathologic immune response to normal resident microbes may result in disease. Fifty percent of patients with Crohn's disease (CD) express antibodies directed against flagellins of the microbiota, with these antibodies associated with stricturing disease [9]. Flagellin antibodies have also been identified in SpA patients, albeit at lower titers [10]. It is unknown whether additional bacteria may be targeted in SpA patients; even in CD, there is evidence of seroreactivity to other antigens of the microbiota [11]. Additionally, Stebbings et al. [8] demonstrated decreased IgA antisera to Bacteroides vulgatus among AS patients, despite increased $\mathrm{T}$ cell proliferative responses to autologous Bacteroides [8]; the same group subsequently demonstrated decreased production of the regulatory cytokine IL-10 by peripheral blood mononuclear cells of AS patients compared to controls [12]. Thus, altered immunologic reactivity to commensal organisms, possibly including Bacteroides, may also play an important role in SpA. Herein, sequencing of the $16 \mathrm{~S}$ ribosomal DNA (rDNA) of patients with enthesitis-related arthritis (ERA; juvenile SpA) and controls was performed, followed by assessment of antigenic reactivity against bacteria differentially present in the patients versus the controls. We hypothesized that ERA patients may have altered microbiota affecting immune responsiveness to enteric microbiota.

\section{Methods}

\section{Patients}

This study was conducted at a single hospital, Children's of Alabama (CoA). SpA was defined according to the International League of Associations for Rheumatology criteria for juvenile idiopathic arthritis subtype, ERA [13]. Controls came from the following sources: 1) children referred to rheumatology clinic, but found to have non-inflammatory causes of joint pain or irrelevant laboratory values (for example, positive antinuclear antibody (ANA) in a well child [14]), and 2) children from the community identified through advertisements or parental affiliation with CoA. Subjects with recent (within 6 months) use of systemic antibiotic therapy were excluded. Clinical, demographic, and laboratory data were obtained through chart review and use of case report forms. Informed consent was obtained from guardians as well as subjects age 14 years and older; assent was obtained from children ages 7 to 13 years. Institutional Review Board approval was obtained from the University of Alabama at Birmingham.

\section{Preparation and sequencing of $16 \mathrm{~S}$ rDNA from fecal material}

Subjects collected the samples at home and immediately placed the samples in a 50-ml container filled with CareyBlair media [15]. Samples were shipped overnight via commercial carrier to our laboratory. Microbial genomic DNA was isolated by standard methods using a kit from Zymo Research (Irvine, CA, USA) as per the manufacturer's instructions. The purified DNA underwent PCR amplification using primers designed to the conserved region flanking the variable IV region from the $16 \mathrm{~S}$ rDNA gene, as per Caporaso et al. [16]. Purification of the resulting PCR product was performed with a Qiagen kit (Valencia, CA, USA) as per the manufacturer's instructions. A second purification with Agencout AmPure beads (Danvers, MA, USA) was performed to increase the quality of the DNA amplicons. The PCR products were multiplexed and diluted for cluster generation using the cBot on a single read v.3 flowcell for the HiSeq2000 (Illumina, San Diego, CA, USA); a few samples were run on the MiSeq (Illumina, San Diego, CA, USA), with the sequences truncated to 101 base pairs to match that of the HiSeq output. Fastq conversion of the raw data files was performed following demultiplexing. Quality control of the fastq files was performed with the FASTX toolkit [17]. The remainder of the steps was performed with the Quantitative Insight into Microbial Ecology (QIIME) suite, version 1.7 [16]. Operational taxonomic units (OTUs) were picked with uclust [18] at 99\% similarity. Taxonomic assignments to OTUs were performed with the Ribosomal Database Project classifier [19], which was trained with the May 2013 version of the Greengenes $16 \mathrm{~S}$ database [20]. Multiple sequence alignment of OTUs was performed with PyNAST [21]. Within-sample (alpha) diversity measures were calculated with Shannon and Phylogenetic Diversity [22], and betadiversity was calculated with Unifrac [23]. Principal coordinates analysis (PCoA) was performed by QIIME to visualize the dissimilarity matrix (beta-diversity) among all the samples, such that samples that are more similar with respect to their taxanomic distribution are closer together in space than samples that are less similar. To assess the statistical significance of the clusters observed from the PCoA, the R package pvclust was used [24]. Pvclust performed hierarchical clustering using the correlation distance matrix; to estimate stability of the resulting clusters, it performed bootstrap analysis at 10,000 iterations, each 
time using different subsets of the original data (with replacement.) The output yields the approximate unbiased (AU) and the bootstrap probability (BP) values of each cluster, with the former said to be less subject to bias as per the original description [24].

\section{ELISA}

Serum samples obtained at approximately the time of the collection of the stool specimens were centrifuged and stored at $-80^{\circ} \mathrm{C}$ until ready for use. ELISA was performed to measure IgA or IgG reactivity in 96-well flat-bottom Costar plates (Fisher, Rockville, MD, USA). In preliminary studies, amounts of lyophilized bacteria antigen and serum dilutions were optimized to identify parameters that permitted reproducible results that differentiated serum samples from negative controls. The optimized amounts of bacterial lysate antigen were $50 \mathrm{ng}$ for Bacteroides fragilis strain 086-5443-2-2 (kindly donated by Cindy Sears), $250 \mathrm{ng}$ for Akkermansia muciniphila (ATCC \# BAA-835), and $100 \mathrm{ng}$ for Faecalibacterium prausnitzii (ATCC \# 27766). Plates were coated with the specified amount of antigen in $50 \mu \mathrm{l}$ of $\mathrm{PBS}$ for four hours at $37^{\circ} \mathrm{C}$, then were transferred to store at $4^{\circ} \mathrm{C}$ overnight. After blocking with $5 \%$ milk, serum was added in duplicate at a 1:50 dilution for two hours at room temperature, followed by the addition of anti-human IgG or IgA conjugated to peroxidase (Sigma, St Louis, MO, USA) at a 1:10,000 dilution overnight at $4^{\circ} \mathrm{C}$. Plates were developed with para-nitrophenyl phosphate and scanned at the $490 \mathrm{~nm}$ wavelength.

\section{Statistical analysis}

Comparisons of individual taxa were performed with the Mann-Whitney $U$-test for two groups and the KruskalWallis test for three groups. Nominal data were evaluated with the chi-squared test. Correlation analyses were performed with the Spearman coefficient. Additionally, IgA and IgG antibodies against B. fragilis and F. prausnitzii were analyzed using a linear model with fecal presence of Bacteriodes and F. prausnitzii, respectively, using diagnosis (patient or control) and the interaction of these two terms as predictors. A two-sided $t$-test was used to test each term for significance, which was set at 0.05 . These analyses were performed with SPSS, Version 17 (Chicago, IL, USA) and R (version 3.0.2.)

\section{Results}

Patients

Clinical and demographic features of the study patients are shown in Table 1. In total 25 children with ERA (11 female, age (median, range) 13,7 to 19 years) and 13 controls (7 female, age 13, 5 to 17 years) were included in the study. Two of the ERA subjects had IBD. Twenty-one
Table 1 Patient population

\begin{tabular}{lll}
\hline Feature & $\begin{array}{l}\text { Enthesitis-related } \\
\text { arthritis }\end{array}$ & Controls \\
\hline Number & 25 & 13 \\
Age, years, median, range & $13.0,7.0$ to 19.0 & $13.0,5.8$ to 18.0 \\
$\begin{array}{l}\text { Male:female, number } \\
\text { Body mass index, } \\
\text { median, range }\end{array}$ & $11: 14$ & $6: 7$ \\
$\begin{array}{l}\text { Disease duration, } \\
\text { years, median, range }\end{array}$ & 20,16 to 41 & $23.7,14.0$ to \\
$\begin{array}{l}\text { HLA-B27+, number } \\
\text { Known inflammatory } \\
\text { bowel disease, number }\end{array}$ & $2.4,0$ to 8.2 & N/A \\
$\begin{array}{l}\text { Erythrocyte sedimentation } \\
\text { rate, median, range }\end{array}$ & $9 / 24(38 \%)$ & $0 / 3$ \\
$\begin{array}{l}\text { Medications } \\
\text { None, number }\end{array}$ & 9,3 to 56 & 0 \\
Methotrexate alone, number & $2(8.0 \%)$ & \\
Pentasa alone, number & $1(4.0 \%)$ & 13 (100\%) \\
Anti-TNF +/- conventional & $15(60 \%)$ & \\
DMARD & & \\
\hline DMARD disease-modifying anti-rheumatic drug (methotrexate, leflunomide)
\end{tabular}

DMARD, disease-modifying anti-rheumatic drug (methotrexate, leflunomide); TNF, tumor necrosis factor; N/A, not applicable.

patients, but no controls, were on immunosuppressive therapy.

\section{Results of $16 \mathrm{~S}$ sequencing analysis}

High-quality sequence data were obtained from fecal samples on all 38 children. Following the filtering step, the sequencing depth ranged from 9,000 to 555,000 , with a sequence length of 101 base pairs. There were no differences in within-subject (alpha) diversity measurements between the groups (not shown). The taxonomic distribution of the top 20 taxa is shown in Figure 1A. Of these, the only two taxa with statistically significant differences favoring the controls were the Faecalibacterium genus (11\% versus 5.2\%, $P=0.005)$ and unspecified genera of the Lachnospiraceae family (12\% versus $7.0 \%$, $P=0.020$ ). Among those favoring ERA, Bifidobacterium showed a modest but statistically significant increase (1.8\% versus $0 \%, P=0.032$ ), and Bacteroides demonstrated a larger but statistically non-significant increase ( $21 \%$ versus $11 \%, P=0.150$ ). Analysis at the species level revealed that $84 \%$ of the Faecalibacterium sequences represented a single species: Faecalibacterium prausnitzii, which constituted $10 \%$ of the microbiota of control patients versus $3.8 \%$ in the ERA group $(P=0.008$.) A basic local alignment search tool (BLAST) search of the OTU representing the majority of the unspecified Lachnospiraceae family members revealed two matches at 100\% identified: Fusicatenibacter saccharivorans and Clostridium clostridioforme. Thus, it is unclear which of 

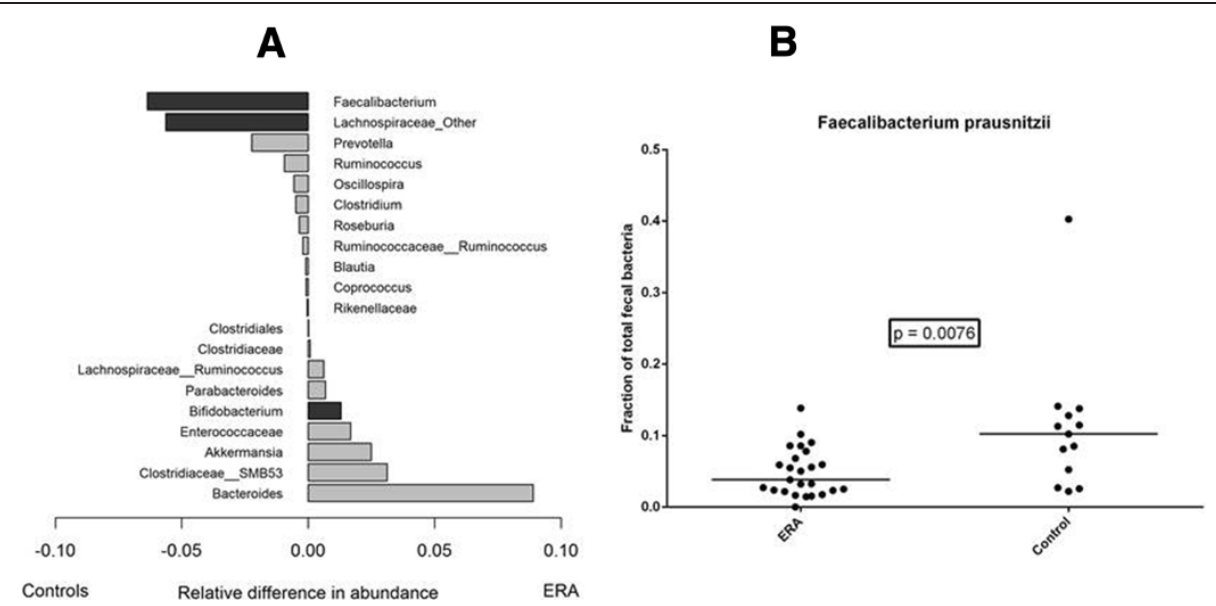

Figure 1 Decreased abundance of Faecalibacterium genus in enthesitis-related arthritis (ERA) patients compared to controls. (A) The relative abundance of the top 20 taxa are shown. Although the taxa were evaluated at the genus level, some of the operational taxonomic units (OTUs) were only identified at the family level or above. Negative values (left) indicate higher frequency in controls, and positive values (right) indicate higher frequencies in patients. Statistically significant differences (Mann-Whitney U-test) are depicted in a darker gray color. (B) The distribution of F. prausnitzii levels is shown.

the two organisms drove this apparent difference. Sixtyseven percent of the Bifidobacterium sequences represented B. adolescentis. The distribution of $F$. prausnitzii levels in patients and controls is depicted in Figure 1B. Additionally, A. muciniphila was present in very low quantities in most patients, but was relatively abundant $(>2 \%)$ in $7 / 25(28 \%)$ patients, but not in any of the 13 controls $(P=0.072$, Fisher's exact). There were no significant associations between the body mass index of the subjects and presence of any of these bacteria (Additional file 1: Table S1.) Among subjects with ERA, HLA-B27 status was not associated with significant differences in the levels of these bacteria (Additional file 1: Table S2).

Thus, at the genus and species level, our data demonstrate some statistically significant differences between patients and controls. Our next step was to assess whether patients and controls may have different overall communities of enteric bacteria. This was evaluated with PCoA (Figure 2A), which demonstrated that while most of the patients clustered with the controls, a set of eight ERA patients formed their own cluster. To confirm the existence of this cluster, we performed bootstrap analysis using pvclust, with the results showing an identical cluster with an AU probability of $99 \%$ (Figure 2B.) Comparing the eight ERA patients in cluster 1 with the remainder of the ERA subjects, the former had significantly higher Bacteroides levels ( $32 \%$ versus $13 \%, P<0.001$ ), with no difference in the $F$. prausnitzii content (4.7\% versus $3.2 \%$, $P=0.897$ ). The subgroup of ERA patients with elevated Bacteroides content appeared to be under-represented among those patients with high levels ( $>2 \%$ ) of Akkermansia, which was found in $0 / 8$ of cluster 1 compared to $7 / 17$
(41\%) of cluster $2(P=0.057)$. Despite the predominance of Bacteroides among the subjects in cluster 1, the Shannon index was similar between the two clusters (Figure 3A). However, cluster 1 demonstrated diminished alpha diversity with the phylogenetic diversity metric (3B). The two clusters otherwise appeared similar with respect to most clinical and laboratory features, with the exception that cluster 1 was predominantly female and cluster 2 predominantly male (Table 2.$)$

Thus, there appear to be significant differences in the populations of enteric bacteria between patients and controls. In order to assess whether the alterations in the frequencies of the bacterial genera could be associated with changes in the immunoreactivity, we performed ELISA against a Bacteroides species (B. fragilis) and F. prausnitzii among the 31 subjects (22 patients and 9 controls) in whom we had serum samples available, including both with IBD. Overall, there were no significant differences in IgA or IgG reactivity against any of the bacteria (Table 3), although HLA-B27+ subjects had elevated antibody production against both (Additional file 1: Table S2.) When broken down by cluster, ERA subjects in cluster 1, characterized by increased bacteroides, also demonstrated increased IgA and IgG reactivity against $B$. fragilis, the latter statistically significant at $P=0.05$ (Additional file 1 : Table S3). Interesting trends emerged when we evaluated antibody production against a particular species as a function of the quantity of the associated bacteria. There was a strong correlation $(r=0.511, P=0.003)$ between Bacteroides content and IgG antibodies against $B$. fragilis among the group as a whole. In contrast, while the overall correlation between Bacteroides content and IgA antibodies was weak $(r=0.300, P=0.067)$, divergent trends 
A

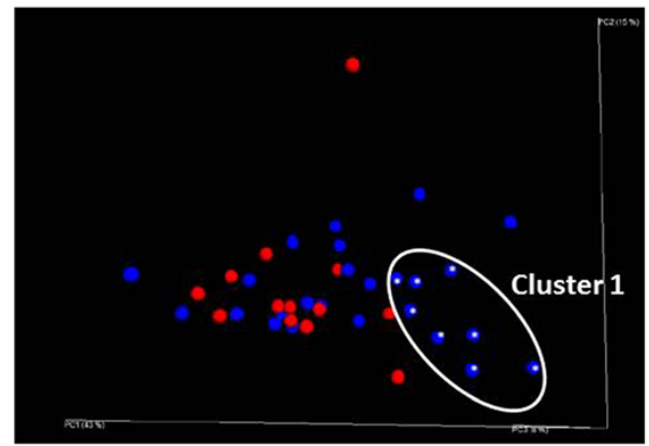

B

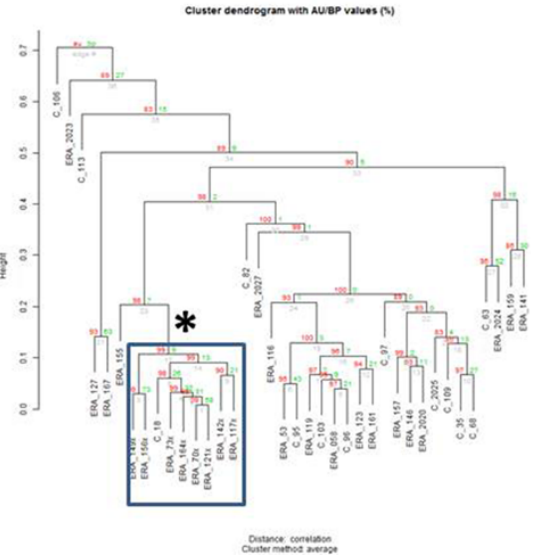

Figure 2 Spondyloarthritis (SpA) patients have two distinct clusters. In principal coordinates analysis (A) each subject is a separate dot; the closer two subjects are in space, the more similar their sequence data. Enthesitis-related arthritis (ERA) subjects are shown in blue, controls in red. The asterisks over eight of the subjects indicate subjects identified as well with the pvclust bootstrap analysis (B). The same eight subjects are present in the identified cluster.

emerged within the two groups (Figure 4): ERA patients demonstrated a similar positive correlation $(r=0.481$, $P=0.005)$, while an inverse although not statistically significant correlation was seen in the control group ( $r=-0.483, P=0.187)$. Linear modeling of the slopes of the two regression lines demonstrated a statistically significant interaction between IgA levels against $B$. fragilis and diagnosis $(P=0.008)$, not present with IgG $(P=0.105)$. A similar discordance was seen with $F$. prausnitzii (Figure 5), although in this case, patients demonstrated an inverse correlation between IgA antibodies to $F$. prausnitzii and F. prausnitzii content $(r=-0.404, P=0.062)$, with controls demonstrating a strong positive correlation $(r=0.850$, $P=0.004)$; as with $B$. fragilis, the slopes of the two curves were statistically significantly different $(P=0.039)$. Neither group demonstrated a strong correlation in either direction between fecal F. prausnitzii content and IgG antibodies to the same, although they trended in the same
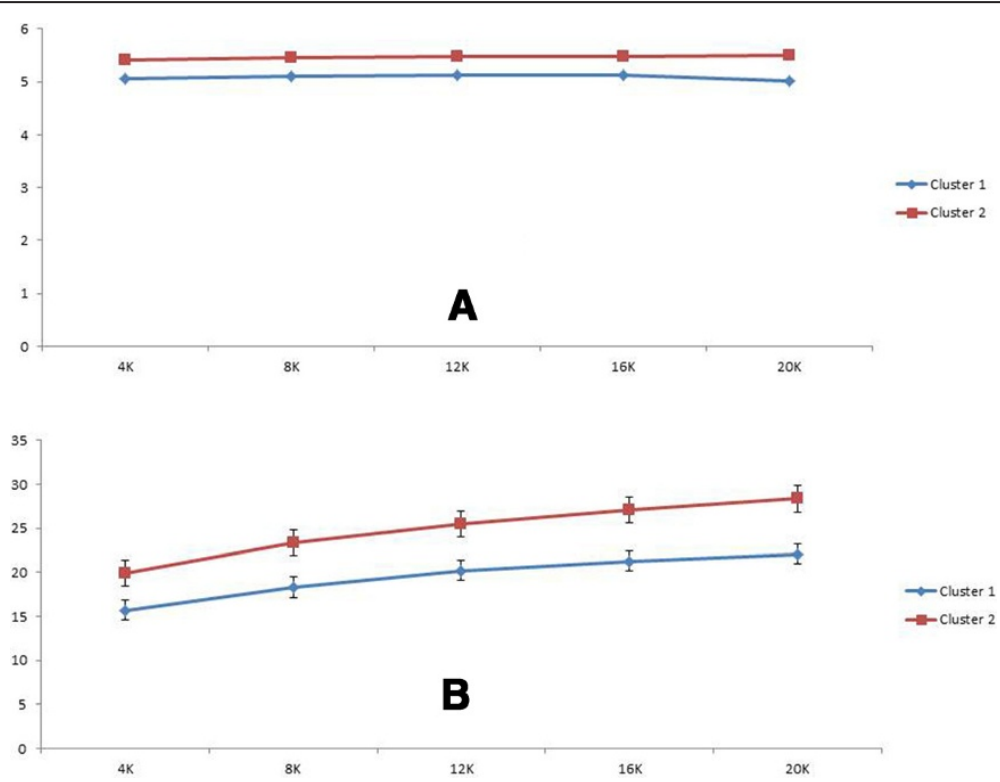

Figure $\mathbf{3}$ Alpha diversity metric comparing clusters $\mathbf{1}$ and $\mathbf{2}$. The Shannon index is shown in $\mathbf{A}$; this measures species evenness, or the probability of being able to predict the identity of a randomly drawn sequence. The phylogenetic diversity is shown in $\mathbf{B}$; this evaluates the length of all branches of the phylogenetic tree of a given population [22]. 
Table 2 Comparison of clinical and laboratory features of children in cluster 1 versus cluster 2

\begin{tabular}{|c|c|c|c|}
\hline Feature & Cluster 1 & Cluster 2 & $P$-value \\
\hline Number & 8 & 17 & N/A \\
\hline Age, years, median, range & 13,7 to 18 & $13,7.5$ to 19 & 0.932 \\
\hline Male:female, number & $1: 7$ & $13: 4$ & 0.007 \\
\hline Body mass index, median, range & 20,16 to 33 & 23,16 to 41 & 0.374 \\
\hline Disease duration, years, median, range & $1.6,0$ to 6.5 & 3,0 to 8.2 & 0.238 \\
\hline HLA-B27+, number & $3 / 8,38 \%$ & $6 / 16,38 \%$ & 1.000 \\
\hline Known inflammatory bowel disease, number & $1,12 \%$ & $1,5.9 \%$ & 1.000 \\
\hline Erythrocyte sedimentation rate, number: median, range & 7: 10,6 to 50 & $13: 9,3$ to 56 & 0.536 \\
\hline \multicolumn{4}{|l|}{ Medications, number } \\
\hline None & $2,25 \%$ & $5,29 \%$ & \\
\hline Methotrexate alone & 0 & $2,12 \%$ & \\
\hline Pentasa alone & 0 & $1,5.9 \%$ & \\
\hline Anti-TNF +/- conventional DMARD & $6,75 \%$ & $9,53 \%$ & \\
\hline
\end{tabular}

DMARD, disease-modifying anti-rheumatic drug; TNF, tumor necrosis factor; N/A, not applicable.

direction (controls: $r=-0.326, P=0.391$; ERA: $r=-0.113$, $P=0.615)$.

\section{Discussion}

The human intestine is colonized with an estimated 100 trillion bacteria, a process that begins shortly after birth [25]. It is becoming increasingly clear that these bacteria play important roles in immune function as well as in a variety of autoimmune and inflammatory disorders [25]. One condition with important microbial contributions is IBD, as evidenced by aberrant microbial content [26], therapeutic response to antibiotics in CD [27], and abnormal innate and adaptive immunologic responses to enteric bacteria [28]. There is also interest in the role of the microbiota in SpA [29], with a previous study showing possible decreases in the Bacteroides-Provotella and Clostridium leptum groups, with an increase in Bifidobacterium among AS patients compared to controls, although the overall differences were not statistically significant [8].

In this study, four candidate organisms are identified as being potentially associated with ERA. Decreased F. prausnitzii, was seen in the patients compared to controls. This finding has previously and consistently been reported in patients with IBD [30-32], and may be of particular significance in light of its reported antiinflammatory effects [33]. It may also be consistent with the previous finding of decreases in the Clostridium leptum group in AS patients [8], as both C. leptum and F. prausnitzii are in the clostridiaceae family. F. prausnitzii is an important producer of butyrate [34], which may have anti-inflammatory properties [35]; there was no compensatory increase in other butyrate-producing bacteria, such as the Eubacterium and Roseburia genera (data not shown) [34,36]. These findings underscore the pathogenic similarities between IBD and ERA, as F. prausnitzii was not reduced in a study of newly diagnosed RA patients [37]. Additional evidence of microbial similarities between IBD and ERA comes from the study by Frank et al. [32], which showed not only decreased F. prausnitzii in the IBD patients, but also decreases in the Lachnospiraceae family. In particular, there may have been a decrease in Clostridium clostridioforme, which was one of the two potential organisms associated with the dominant OTU in the Lachnospiraceae family significantly under-represented in the ERA patients. That these differences may result from genetic differences between the populations, rather than from the disease

Table 3 Median antibody levels (o.d.) against several of the bacteria differentially present between patients and controls

\begin{tabular}{|c|c|c|c|c|c|c|}
\hline \multirow[t]{2}{*}{ Bacteria } & \multicolumn{3}{|l|}{$\lg A$} & \multicolumn{3}{|l|}{$\lg G$} \\
\hline & ERA & Control & $P$-value & ERA & Control & $P$-value \\
\hline \multirow[t]{2}{*}{ B. fragilis } & 0.11 & 0.16 & 0.094 & 0.41 & 0.43 & 0.536 \\
\hline & 0 to 0.21 & 0.094 to 0.343 & & 0.095 to 1.3 & 0.20 to 1.6 & \\
\hline \multirow[t]{2}{*}{ F. prausnitzii } & 0.15 & 0.18 & 0.112 & 0.45 & 0.46 & 0.742 \\
\hline & 0.043 to 0.51 & 0.14 to 0.29 & & 0.21 to 1.6 & 0.22 to 1.6 & \\
\hline
\end{tabular}

ERA, enthesitis-related arthritis. Values are median (min - max). o.d. = optical density. 


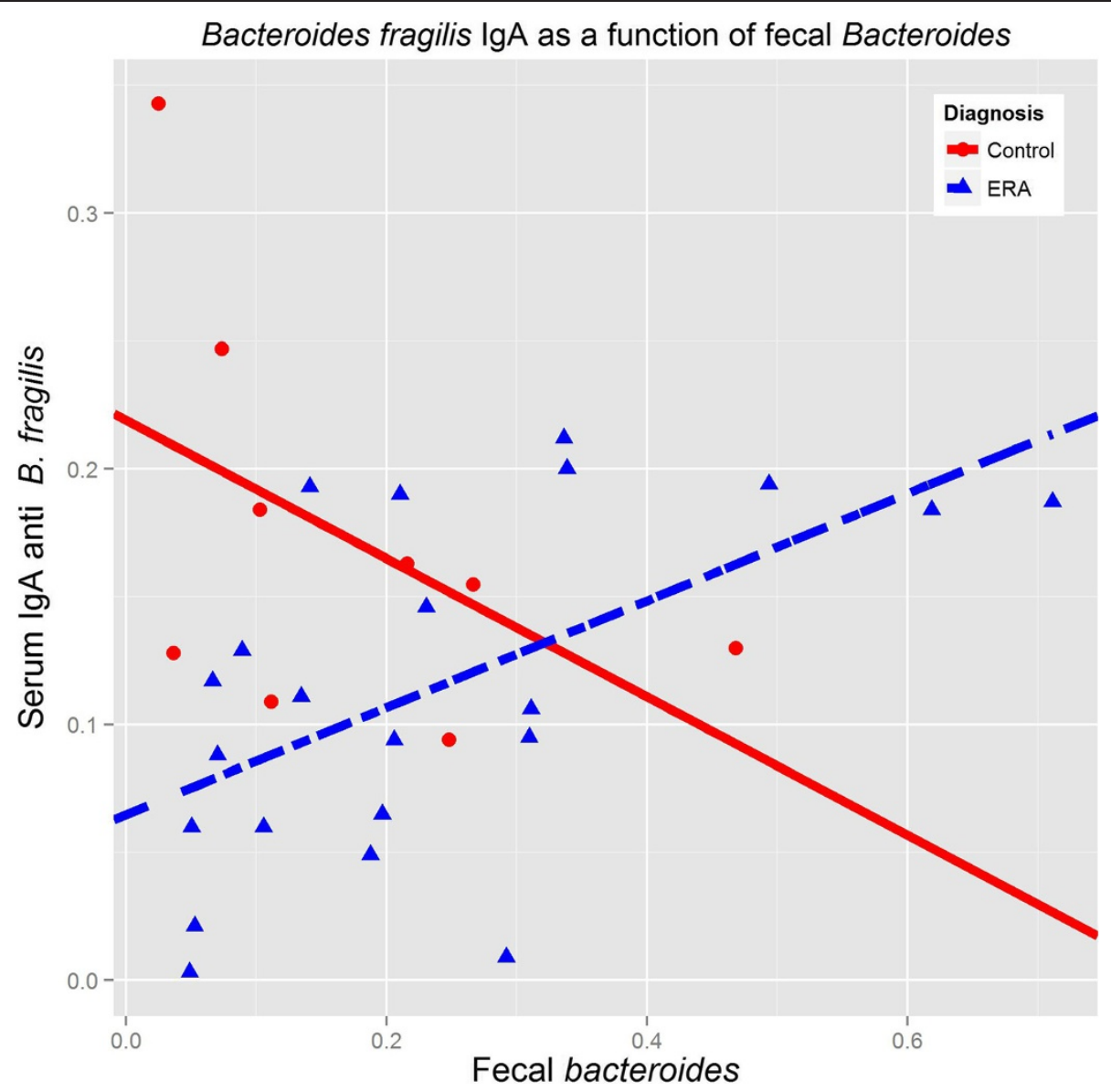

Figure 4 Divergent relationships between $B$. fragilis $\lg A$ levels and fecal Bacteroides content, between patients and controls.

Enthesitis-related arthritis (ERA) subjects are depicted in blue (broken line); controls are red (solid line).

process itself, was suggested by a study showing that NOD2 and ATG16L1 genotypes were associated with altered microbial populations even with adjustment for diagnosis of IBD [38]. This information was not available for our patients.

A novel finding of this study is that both Bacteroides species and Akkermansia muciniphila were found to be associated with disease states in largely non-overlapping subsets of ERA patients. These subsets were similar with respect to most clinical and laboratory features, aside from cluster 1 being predominantly female, and cluster 2 predominantly male. The potential relevance of this distinction awaits further exploration. B. fragilis is considered to have regulatory effects through its polysaccharide A [39], although the enterotoxigenic strain of $B$. fragilis has been linked to IBD [40]. Additionally, in both a rat and a mouse model of SpA abrogated by the germ-free state, addition of a consortium of bacteria that includes bacteroides species results in active disease, with $B$. vulgatus specifically identified as a causative agent in the rat model [41,42]. Furthermore, as indicated above, abnormal humoral and $\mathrm{T}$ cell responses to bacteroides has been reported in AS patients $[8,12]$. The data reported herein indicated that up to one third of ERA patients have markedly elevated Bacteroides content, while a second subset had similar overall patterns of enteric bacteria to the controls, with the exception of lower Faecalibacterium. However, 7/17 of the ERA group with normal Bacteroides content, compared to 0/8 of the group with elevated Bacteroides content, carried increased frequencies of $A$. muciniphila. First described in 2004, it was originally isolated based upon its capacity to thrive on intestinal mucin, suggesting that high quantities could potentially lead to a defect in the intestinal wall barrier function [43]. Although a European study indicated that $A$. muciniphila typically comprises 1 to $3 \%$ of the intestinal microbiota [44], it is evidently less common in the United States, or at least in our patient population, and there may be regional differences in the overall microbiota that could result in patients in this region being more susceptible to a pro-inflammatory effect of this bacterium. This is the first study suggesting a potential role in inflammatory disease in humans, although a recent study of mice infected with Salmonella demonstrated increased histologic inflammatory scores and mRNA levels of 


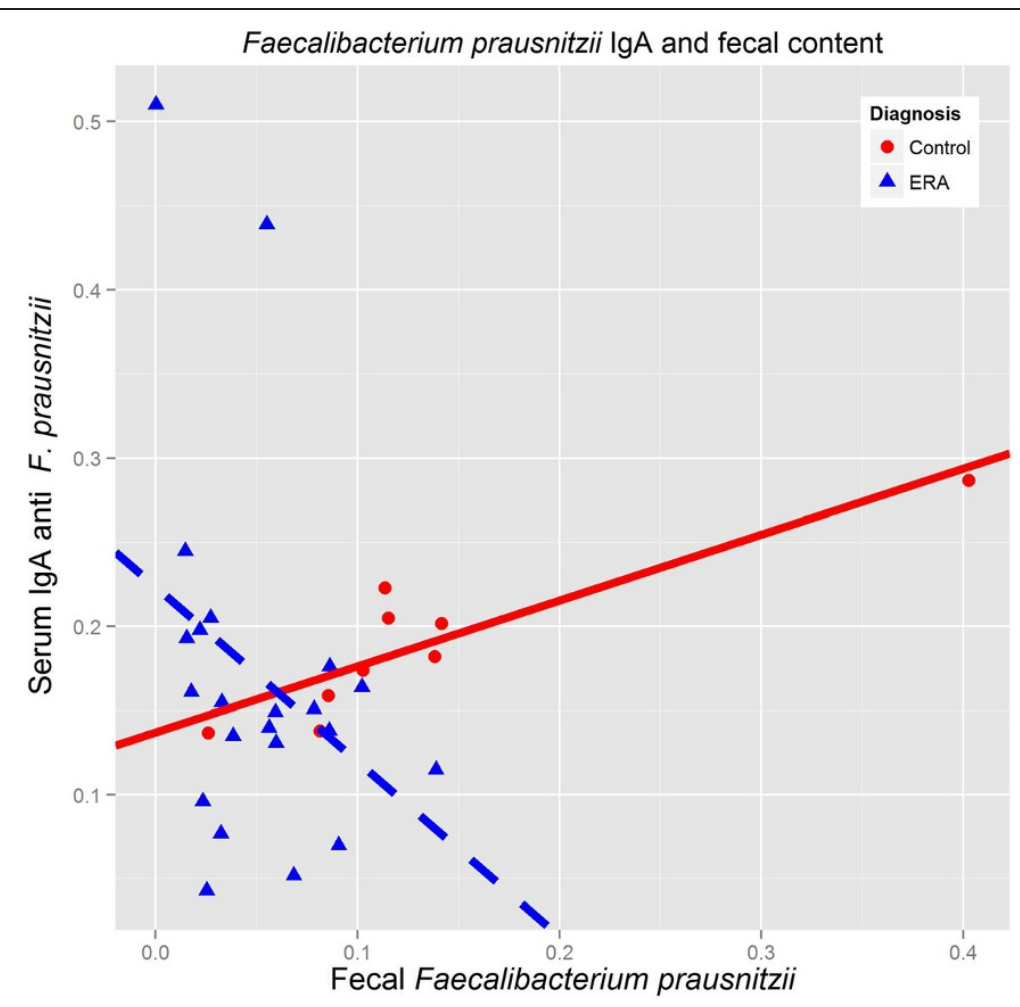

Figure 5 Divergent relationships between F. prausnitzii IgA levels and fecal F. prausnitzii content, between patients and controls. Enthesitis-related arthritis (ERA) subjects are depicted in blue (broken line); controls are red (solid line).

inflammatory markers in mice that also received $A$. muciniphila [45].

Finally, elevated Bifidobacterium, mostly B. adolescentis, was also identified among ERA patients. There are mixed data with respect to this genus in the context of IBD, with prior studies both showing elevated [31] as well as decreased presence [46]. Elevated bifidobacterium was also seen in the study of AS patients [8]. Thus, overall, ERA patients displayed a microbiota profile similar to that published for IBD patients with respect to lower levels of F. prausnitzii and possibly of Clostridium clostridioforme, as well as alterations in B. adolescentis. Our findings are also consistent with those reported in AS patients, despite substantial differences in age and disease duration in the current study compared to the previous [8].

IgA functions in part to protect the host against commensal organisms $[47,48]$. It also has a regulatory role, with downstream responses to IgA binding dependent upon a variety of factors, including signaling events through the IgA receptor and the location of the dendritic antigen presenting cells $[49,50]$. Thus, the interpretation of discordant correlations between IgA and $16 \mathrm{~S}$ levels between patients and controls is clouded by the multiple functions of IgA. In the case of $B$. fragilis, the relationship between the IgA and the sequence data may represent invasion of the gut wall by the bacterium, triggering systemic immune responses. Alternatively, in light of its potential regulatory role, the inverse relationship between IgA against $F$. prausnitzii and the sequence data may represent a failure of the system to generate a regulatory $\mathrm{T}$ cell response normally thought to be induced by this species [33]. Studies of $\mathrm{T}$ cell function are required to evaluate these possibilities.

This study has several limitations. The patient numbers are limited, and they included subjects with newly diagnosed as well as treated arthritis. However, a study of IBD patients showed that while antibiotics and aminosalicylates had important effects on the microbiota, immunosuppressive therapies appeared to have less of an impact [51]. Exclusion of patients with IBD did not impact the trends shown herein, although the association between F. prausntzii levels and antibodies among ERA patients was less robust (Additional file 1: Tables S4, S5).

An additional limitation is that information on the diet of these subjects was unavailable on most of the patients. However, body mass index data were not predictive of the levels of any of the bacteria identified in this study (Additional file 1: Table S1). Furthermore, although the controls did have higher BMI compared to the patients (Table 1), at the phylum level, the Firmicutes: Bacteroidetes ratio was similar (2.97, 0.26 to 30 in ERA patients compared to $3.46,1.0$ to 26 in controls, $P=0.199$, 
and there was a small albeit statistically insignificant inverse relationship between body mass index and the Firmicutes/Bacteroidetes ratio among the group as a whole ( $r=-0.140, P=0.401$ ), the opposite direction that would be expected if weight were the driving factor [52]. Finally, there was no external validation of the differences in bacterial content observed in this study, nor was there objective measurement of intestinal inflammation (for example, fecal calprotectin.) This is, nevertheless, the first comprehensive evaluation of the microbiota in pediatric or adult $\mathrm{SpA}$, confirming a potential role for insufficient protective F. prausnitzii in the pathogenesis of ERA and introducing potential novel bacteria as associative agents in ERA. These findings suggest altering the gut microbiota may be beneficial in children with ERA. Future directions will extend these findings in larger cohorts and also identify the nature of the adaptive (antibody and $\mathrm{T}$ cell reactivity) immunologic response against these enteric commensal organisms.

\section{Conclusions}

Our studies provide further evidence of a pathogenetic link between IBD and SpA by showing a similar decrease in fecal F. prausnitzii content in ERA patients as had previously been reported in subjects with IBD. In addition, we introduce two organisms, Bacteroides genus and $A$. muciniphila, as potential associative agents in subsets of children with ERA. Finally, our data suggest that alterations to IgA reactivities against commensal organisms may play a role in ERA.

\section{Additional file}

Additional file 1: Table S1. Correlation between body mass index and sequencing data. Table S2. Effect of HLA-B27 status on sequencing data and antibody titers, among subjects with enthesitis-related arthritis (ERA). Table S3. Antibody titers by ERA cluster. Table S4. Sequencing data and antibodies titers, following exclusion of patients with inflammatory bowel disease (IBD). Table S5. Correlation between sequencing data and antibody titers, following exclusion of subjects with IBD.

\section{Abbreviations \\ AS: ankylosing spondylitis; AU: approximate unbiased; BMI: body mass index; BP: bootstrap probability; CD: Crohn's disease; CoA: Children's of Alabama: DMARD: disease-modifying anti-rheumatic drug; ELISA: enzyme-linked immunosorbent assay; ERA: enthesitis-related arthritis; IBD: inflammatory bowel disease; OTU: operational taxonomic unit; PBS: phosphate-buffered saline; PCOA: principal coordinates analysis; QIIME: Quantitative Insight Into Microbial Ecology; RA: rheumatoid arthritis; rDNA: ribosomal DNA; SpA: spondyloarthritis; TNF: tumor necrosis factor.}

\section{Competing interests}

The authors declare that they have no competing interests.

\section{Authors' contributions}

MLS helped design the study, performed some of the analyses, and wrote the first draft of the manuscript. RK and EJL contributed to the $16 \mathrm{~S}$ analysis. CDM helped design the study, assisted with interpretation of the data and performed the PCR amplification of the 16S DNA. XC assisted with the cluster analysis and wrote the code for the linear modeling. AG performed the ELISA. RQC and COE helped design the study and assisted with interpretation of the laboratory results. All authors critically revised the manuscript drafts and approved the final version of the manuscript.

\section{Acknowledgements}

The authors wish to acknowledge Dr Peter Eipers for sample preparation. This work was supported by an American College of Rheumatology, Rheumatology Research Foundation Investigator Award (to MLS), NIH/NIAMS P60 AR064172 (to COE), and internal funding from Children's of Alabama (to MLS). The following are acknowledged for their support of the Microbiome Resource at the University of Alabama at Birmingham: School of Medicine, Comprehensive Cancer Center (P30 AR050948), Center for AIDS Research (5P30 Al027767), Center for Clinical Translational Science (UL1 TR000165) and the Heflin Center. There are no commercial supporters of this work, and none of the authors have any financial conflicts of interest.

\section{Author details}

'Department of Pediatric Rheumatology, University of Alabama at Birmingham, CPP N 210M, 1600 7th Avenue South, Birmingham, AL 35233, USA. ${ }^{2}$ Center for Clinical and Translational Sciences, University of Alabama at Birmingham, CPP N 210M, 1720 2nd Avenue South, Birmingham, AL 35294, USA. ${ }^{3}$ Cell, Developmental, and Integrative Biology, University of Alabama at Birmingham, CPP N 210M, 1720 2nd Avenue South, Birmingham, AL 35294, USA. ${ }^{4}$ Department of Microbiology, University of Alabama at Birmingham, CPP N 210M, 1720 2nd Avenue South, Birmingham, AL 35294, USA. ${ }^{5}$ Department of Biostatistics, University of Alabama at Birmingham, CPP N 210M, 1720 2nd Avenue South, Birmingham, AL 35294, USA. ${ }^{6}$ Department of Medicine - Gastroenterology, University of Alabama at Birmingham, CPP N 210M, 1720 2nd Avenue South, Birmingham, AL 35294, USA.

Received: 8 July 2014 Accepted: 10 November 2014

Published online: 30 November 2014

\section{References}

1. Helmick CG, Felson DT, Lawrence RC, Gabriel S, Hirsch R, Kwoh CK, Liang MH, Kremers HM, Mayes MD, Merkel PA, Pillemer SR, Reveille JD, Stone JH, National Arthritis Data Workgroup: Estimates of the prevalence of arthritis and other rheumatic conditions in the United States. Part I. Arthritis Rheum 2008, 58:15-25.

2. Reveille JD: The genetic basis of spondyloarthritis. Ann Rheum Dis 2011, 70:i44-i50.

3. Reveille JD: Genetics of spondyloarthritis-beyond the MHC. Nat Rev Rheumatol 2012, 8:296-304.

4. Fantini MC, Pallone F, Monteleone G: Common immunologic mechanisms in inflammatory bowel disease and spondylarthropathies. World J Gastroenterol 2009, 15:2472-2478.

5. Taurog JD, Richardson JA, Croft JT, Simmons WA, Zhou M, Fernandez-Sueiro $J$, Balish E, Hammer RE: The germfree state prevents development of gut and joint inflammatory disease in HLA-B27 transgenic rats. J Exp Med 1994, 180:2359-2364.

6. Metzker ML: Sequencing technologies - the next generation. Nat Rev Genet 2010, 11:31-46.

7. Cheng J, Palva AM, de Vos WM, Satokari R: Contribution of the intestinal microbiota to human health: from birth to 100 years of age. Curr Top Microbiol Immunol 2013, 358:323-346.

8. Stebbings S, Munro K, Simon MA, Tannock G, Highton J, Harmsen H, Welling G, Seksik P, Dore J, Grame G, Tilsala-Timisjarvi A: Comparison of the faecal microflora of patients with ankylosing spondylitis and controls using molecular methods of analysis. Rheumatology (Oxford) 2002, 41:1395-1401

9. Targan SR, Landers CJ, Yang H, Lodes MJ, Cong Y, Papadakis KA, Vasiliauskas E, Elson CO, Hershberg RM: Antibodies to CBir1 flagellin define a unique response that is associated independently with complicated Crohn's disease. Gastroenterology 2005, 128:2020-2028

10. Wallis D, Asaduzzaman A, Weisman M, Haroon N, Anton A, McGovern D, Targan S, Inman R: Elevated serum anti-flagellin antibodies implicate subclinical bowel inflammation in ankylosing spondylitis: an observational study. Arthritis Res Ther 2013, 15:R166.

11. Lodes MJ, Cong Y, Elson CO, Mohamath R, Landers CJ, Targan SR, Fort M, Hershberg RM: Bacterial flagellin is a dominant antigen in Crohn disease. J Clin Invest 2004, 113:1296-1306. 
12. Stebbings SM, Taylor C, Tannock GW, Baird MA, Highton J: The immune response to autologous bacteroides in ankylosing spondylitis is characterized by reduced interleukin 10 production. J Rheumatol 2009, 36:797-800.

13. Petty RE, Southwood TR, Manners P, Baum J, Glass DN, Goldenberg J, He X, Maldonado-Cocco J, Orozco-Alcala J, Prieur AM, Suarez-Almazor ME, Woo P: International League of Associations for Rheumatology classification of juvenile idiopathic arthritis: second revision, Edmonton, 2001. J Rheumatol 2004, 31:390-392.

14. McGhee JL, Burks FN, Sheckels $J$, Jarvis JN: Identifying children with chronic arthritis based on chief complaints: absence of predictive value for musculoskeletal pain as an indicator of rheumatic disease in children. Pediatrics 2002, 110:354-359.

15. Cary SG, Blair EB: New Transport Medium for Shipment of Clinical Specimens. I. Fecal Specimens. J Bacterio/ 1964, 88:96-98.

16. Caporaso JG, Lauber CL, Walters WA, Berg-Lyons D, Lozupone CA, Turnbaugh PJ, Fierer N, Knight R: Global patterns of $16 \mathrm{~S}$ rRNA diversity at a depth of millions of sequences per sample. Proc Natl Acad Sci U S A 2011, 108:4516-4522.

17. Gordon A, Hannon GJ: "FASTX-Toolkit", FASTQ/A short-reads pre-processing tools (unpublished). 2013. Available from: http://hannonlab.cshl.edu/fastx toolkit/.

18. Edgar RC: Search and clustering orders of magnitude faster than BLAST. Bioinformatics 2010, 26:2460-2461.

19. Wang Q, Garrity GM, Tiedje JM, Cole JR: Naive Bayesian classifier for rapid assignment of rRNA sequences into the new bacterial taxonomy. Appl Environ Microbiol 2007, 73:5261-5267.

20. DeSantis TZ, Hugenholtz P, Larsen N, Rojas M, Brodie EL, Keller K, Huber T, Dalevi D, Hu P, Andersen GL: Greengenes, a chimera-checked 16S rRNA gene database and workbench compatible with ARB. Appl Environ Microbiol 2006, 72:5069-5072

21. Caporaso JG, Bittinger K, Bushman FD, DeSantis TZ, Andersen GL, Knight R: PyNAST: a flexible tool for aligning sequences to a template alignment. Bioinformatics 2010, 26:266-267.

22. Faith DP, Baker AM: Phylogenetic diversity (PD) and biodiversity conservation: some bioinformatics challenges. Evol Bioinform Online 2006, 2:121-128.

23. Lozupone $C$, Knight R: UniFrac: a new phylogenetic method for comparing microbial communities. Appl Environ Microbio/ 2005, 71:8228-8235.

24. Suzuki R, Shimodaira H: Pvclust: an R package for assessing the uncertainty in hierarchical clustering. Bioinformatics 2006, 22:1540-1542.

25. Johnson CL, Versalovic J: The human microbiome and its potential importance to pediatrics. Pediatrics 2012, 129:950-960.

26. De Cruz P, Prideaux L, Wagner J, Ng SC, McSweeney C, Kirkwood C, Morrison M, Kamm MA: Characterization of the gastrointestinal microbiota in health and inflammatory bowel disease. Inflamm Bowel Dis 2012, 18:372-390.

27. Sartor RB: Therapeutic manipulation of the enteric microflora in inflammatory bowel diseases: antibiotics, probiotics, and prebiotics. Gastroenterology 2004, 126:1620-1633.

28. Elson CO, Cong Y: Host-microbiota interactions in inflammatory bowe disease. Gut Microbes 2012, 3:332-344.

29. Rosenbaum JT, Davey MP: Time for a gut check: evidence for the hypothesis that HLA-B27 predisposes to ankylosing spondylitis by altering the microbiome. Arthritis Rheum 2011, 63:3195-3198.

30. Thorkildsen LT, Nwosu FC, Avershina E, Ricanek P, Perminow G, Brackmann $\mathrm{S}$, Vatn $\mathrm{MH}$, Rudi K: Dominant fecal microbiota in newly diagnosed untreated inflammatory bowel disease patients. Gastroenterol Res Pract 2013, 2013:636785.

31. Wang W, Chen L, Zhou R, Wang X, Song L, Huang S, Wang G, Xia B: Increased proportions of bifidobacterium and the lactobacillus group and loss of butyrate-producing bacteria in inflammatory bowel disease J Clin Microbiol 2014, 52:398-406.

32. Frank DN, St Amand AL, Feldman RA, Boedeker EC, Harpaz N, Pace NR: Molecular-phylogenetic characterization of microbial community imbalances in human inflammatory bowel diseases. Proc Natl Acad SC U S A 2007, 104:13780-13785.

33. Sokol H, Pigneur B, Watterlot L, Lakhdari O, Bermudez-Humaran LG, Gratadoux J, Blugeon S, Bridonneau C, Furet JP, Corthier G, Grangette C, Vasquez N, Pochart P, Trugnan G, Thomas G, Blottière HM, Doré J, Marteau P, Seksik P, Langella P: Faecalibacterium prausnitzii is an anti-inflammatory commensal bacterium identified by gut microbiota analysis of Crohn disease patients. Proc Natl Acad Sci U S A 2008, 105:16731-16736.

34. Hold GL, Schwiertz A, Aminov Rl, Blaut M, Flint HJ: Oligonucleotide probes that detect quantitatively significant groups of butyrate-producing bacteria in human feces. Appl Environ Microbiol 2003, 69:4320-4324.

35. Segain JP, Raingeard de la Blétière $D$, Bourreille A, Leray $V$, Gervois $N$, Rosales C, Ferrier L, Bonnet C, Blottière HM, Galmiche JP: Butyrate inhibits inflammatory responses through NFkappaB inhibition: implications for Crohn's disease. Gut 2000, 47:397-403.

36. Louis P, Young P, Holtrop G, Flint HJ: Diversity of human colonic butyrateproducing bacteria revealed by analysis of the butyryl-CoA:acetate CoA-transferase gene. Environ Microbiol 2010, 12:304-314.

37. Scher JU, Sczesnak A, Longman RS, Segata N, Ubeda C, Bielski C, Rostron T, Cerundolo V, Pamer EG, Abramson SB, Huttenhower C, Littman DR: Expansion of intestinal Prevotella copri correlates with enhanced susceptibility to arthritis. Elife 2013, 2:e01202.

38. Frank DN, Robertson CE, Hamm CM, Kpadeh Z, Zhang $T$, Chen $H$, Zhu W, Sartor RB, Boedeker EC, Harpaz N, Pace NR, Li E: Disease phenotype and genotype are associated with shifts in intestinal-associated microbiota in inflammatory bowel diseases. Inflamm Bowel Dis 2011, 17:179-184.

39. Round JL, Lee SM, Li J, Tran G, Jabri B, Chatila TA, Mazmanian SK: The Toll-like receptor 2 pathway establishes colonization by a commensal of the human microbiota. Science 2011, 332:974-977.

40. Prindiville TP, Sheikh RA, Cohen SH, Tang YJ, Cantrell MC, Silva J Jr: Bacteroides fragilis enterotoxin gene sequences in patients with inflammatory bowel disease. Emerg Infect Dis 2000, 6:171-174.

41. Sinkorova Z, Capkova J, Niederlova J, Stepankova R, Sinkora J: Commensal intestinal bacterial strains trigger ankylosing enthesopathy of the ankle in inbred B10.BR (H-2(k)) male mice. Hum Immunol 2008, 69:845-850.

42. Rath HC, Herfarth HH, Ikeda JS, Grenther WB, Hamm TE Jr, Balish E, Taurog JD, Hammer RE, Wilson KH, Sartor RB: Normal luminal bacteria, especially Bacteroides species, mediate chronic colitis, gastritis, and arthritis in HLA-B27/human beta2 microglobulin transgenic rats. J Clin Invest 1996, 98:945-953.

43. Derrien M, Vaughan EE, Plugge CM, de Vos WM: Akkermansia muciniphila gen. nov., sp. nov., a human intestinal mucin-degrading bacterium. Int J Syst Evol Microbiol 2004, 54:1469-1476.

44. Derrien M, Collado MC, Ben-Amor K, Salminen S, de Vos WM: The Mucin degrader Akkermansia muciniphila is an abundant resident of the human intestinal tract. Appl Environ Microbiol 2008, 74:1646-1648.

45. Ganesh BP, Klopfleisch R, Loh G, Blaut M: Commensal Akkermansia muciniphila exacerbates gut inflammation in Salmonella Typhimuriuminfected gnotobiotic mice. PLoS One 2013, 8:e74963

46. Fite A, Macfarlane S, Furrie E, Bahrami B, Cummings JH, Steinke DT, MacFarlane GT: Longitudinal analyses of gut mucosal microbiotas in ulcerative colitis in relation to patient age and disease severity and duration. J Clin Microbiol 2013, 51:849-856.

47. Macpherson AJ, McCoy KD, Johansen FE, Brandtzaeg P: The immune geography of IgA induction and function. Mucosal Immunol 2008, 1:11-22.

48. Gutzeit C, Magri G, Cerutti A: Intestinal IgA production and its role in host-microbe interaction. Immunol Rev 2014, 260:76-85.

49. Liu LM, MacPherson GG: Rat intestinal dendritic cells: immunostimulatory potency and phenotypic characterization. Immunology 1995, 85:88-93.

50. Wu J, Ji C, Xie F, Langefeld CD, Qian K, Gibson AW, Edberg JC, Kimberly RP: FcalphaRI (CD89) alleles determine the proinflammatory potential of serum IgA. J Immunol 2007, 178:3973-3982.

51. Morgan XC, Tickle TL, Sokol H, Gevers D, Devaney KL, Ward DV, Reyes JA, Shah SA, LeLeiko N, Snapper SB, Bousvaros A, Korzenik J, Sands BE, Xavier $R J$, Huttenhower $C$ : Dysfunction of the intestinal microbiome in inflammatory bowel disease and treatment. Genome Biol 2012, 13:R79.

52. Turnbaugh PJ, Ley RE, Mahowald MA, Magrini V, Mardis ER, Gordon J: An obesity-associated gut microbiome with increased capacity for energy harvest. Nature 2006, 444:1027-1031.

doi:10.1186/s13075-014-0486-0

Cite this article as: Stoll et al:: Altered microbiota associated with abnormal humoral immune responses to commensal organisms in enthesitis-related arthritis. Arthritis Research \& Therapy 2014 16:486. 